INJE-TP-00-04

\title{
Stability of the GRS Model
}

\author{
Y.S. Myung* and Gungwon Kang ${ }^{\dagger}$ \\ Department of Physics, Inje University, Kimhae 621-749, Korea
}

\begin{abstract}
We discuss the compatibility between the weaker energy condition and the stability of Gregory, Rubakov and Sibiryakov (GRS) model. Because the GRS spacetime violates the weak energy condition, it may cause the instability. In the GRS model, the four dimensional gravity can be described by the massive KK modes with the resonance. Hence, instead of considering the weaker energy condition, we require for the stability of this model: no tachyon and no ghost condition for graviton modes $\left(h_{\mu \nu}\right)$. No tachyonic condition $\left(m_{h}^{2} \geq 0\right)$ is satisfied because the lowest state $m_{h}=0$ is supersymmetric vacuum state. Further, no ghost state condition is achieved if one requires some relations for the matter source: $2 T_{55}=T_{\mu}^{\mu}=3\left(T_{22}+T_{33}\right)$. It turns out that, although the GRS spacetime does not satisfy the weaker energy condition, it is stable against small perturbation.
\end{abstract}

${ }^{*}$ E-mail address: ysmyung@physics.inje.ac.kr

${ }^{\dagger}$ E-mail address: kang@physics.inje.ac.kr 


\section{INTRODUCTION}

Recently, there have been lots of interest in the phenomenon of localization of gravity proposed by Randall and Sundrum (RS) [1] (for previous relevant work see references therein). RS assumed a single positive tension 3-brane and a negative bulk cosmological constant in the five dimensional (5D) spacetime. By considering metric fluctuations from a background which is isomorphic to sections of 5D anti-de Sitter spacetime $\left(A d S_{5}\right)$, they have shown that it reproduces the effect of four dimensional (4D) gravity localized on the brane without the need to compactify the extra dimension due to the "warping" in the fifth dimensional space. In more detail, the solution to linearized equations in the five dimensions results in a zero mode, which can be identified with the $4 \mathrm{D}$ massless graviton, and massive continuum Kaluza-Klein (KK) modes. Surprisingly, the wavefunctions of the massive continuum KK modes are suppressed at the brane for small energies, and thus ordinary gravity localized on the brane is reproduced at large distances.

Gregory, Rubakov and Sibiryakov (GRS) [2] have recently considered a brane model which is not asymptotically $A d S_{5}$, but Minkowski flat. In the GRS model, the ordinary 4D Newtonian potential is reproduced from not the massless zero mode, but the resonance of zero mass in the continuum KK spectrum [2]5]. In this sense the GRS model of "a resonance graviton" would differ from the RS model. However, there exist two potential problems with this model: One is the mismatch in polarization states and the other is the violation of weaker energy condition (WEC). It was pointed out that a massive graviton propagator with 3 polarization states does not reproduce the massless graviton propagator with 2 due to the missmatch of the number of polarization states [四. Contrary to it, Csáki, Erlich and Hollowood [6] have argued that in the presence of localized source at $y=0$ the effect $\left(\xi^{5}\right)$ of the bending of the brane exactly compensates for the extra polarization in the massive graviton propagator. Thus the $m_{h}^{2} \rightarrow 0$ limit of the massive propagator at intermediate scales is equivalent to the massless propagator of the Einstein theory just as in the RS scenario. At ultra large scales, however, this theory includes scalar anti-gravity [7], which may be cured by the RG analysis [8]. The most important fact is probably that the mechanism to cancel the extra polarization gives arise to the ghost problem [9]. In fact, the role of the radion field with a negative kinetic term is discussed in models with metastable graviton [10]. In order to have a well-defined theory, the ghost should disappear.

We have introduced the trace field $(h)$ in the RS model [11] instead of $\xi^{5}$ in Ref. [12]. Instead of the localized source $\left(T_{\mu \nu}(x, y)=T_{\mu \nu}(x) \delta(y)\right)$, we introduce a matter source with uniform trace along the extra dimension $\left(T_{\mu}^{\mu}(x, y)=T_{\mu}^{\mu}(x)\right)$. Fortunately, it is shown that massive graviton modes contain ghost states which can be removed by assuming a further condition on the matter source.

The WEC is a basic requirement. In the GRS model we find that this is violated. But the compatibility between the WEC and the recovery of Einstein gravity seems to be not so important. In the brane world, the first thing that we have to do is to recover the Einstein gravity. A more important thing is that the weak energy condition may be closely related to the stability of the GRS spacetime. However, the actual stability analysis of a nonlinear system of the GRS model means to assess the reliability of its linearized approximation [13.

In this paper we wish to study the stability of the GRS background. We require that the stability of this spacetime be given by two: 
1. There are no tachyons for $h_{\mu \nu}(x)$.

2. $h_{\mu \nu}(x)$ has no ghost (i.e., no negative norm state).

We will show that although the GRS spacetime does not satisfy the WEC, this spacetime is stable. In this paper, we use the signature $(-,+,+,+,+)$ and MTW conventions.

\section{WEAKER ENERGY CONDITION (WEC)}

In this section we explicitly show that the GRS model does not satisfy the weakest form of a positive energy condition, which is the so-called null energy condition or the weaker energy condition, saying that the stress-energy tensor $T_{M N}$ obeys $T_{M N} \xi^{M} \xi^{N} \geq 0$ for any null vector $\xi^{M}$. As pointed out by Witten [5], given such energy condition, a "holographic $c$ theorem" for the $A d S_{5}$ says that as one approaches to spatial infinity in the extra dimension, the bulk cosmological constant $\Lambda$ can only become more negative. The bulk cosmological constant in the GRS model is a negative constant in the vicinity of the positive tension brane at the center, but vanishes beyond the negative tension branes.

Let us consider a five-dimensional spacetime which is described by the metric as

$$
d s^{2}=\hat{g}_{M N} d x^{M} d x^{N}=e^{2 A(y)} g_{\mu \nu} d x^{\mu} d x^{\nu}+d y^{2},
$$

where $g_{\mu \nu}$ is Ricci flat (i.e., $R_{\mu \nu}(g)=0$ ) and we assume that the background matter $\left(T_{M N}^{(0)}\right)$ producing such metric is distributed only on four-dimensional domain walls (i.e., $T_{y y}^{(0)}=0$ ). Then the non-vanishing components of the Ricci tensor are [14]

$$
\hat{R}_{\mu \nu}=-\left[A^{\prime \prime}+4\left(A^{\prime}\right)^{2}\right] \hat{g}_{\mu \nu}, \quad \hat{R}_{y y}=-4\left[A^{\prime \prime}+\left(A^{\prime}\right)^{2}\right] .
$$

Here $A^{\prime}=\partial_{y} A$. Note that the contribution to the background matter stress tensor coming from the cosmological constant does not affect the WEC because it is proportional to the

metric. Hence we separate $\Lambda$ from $T_{M N}^{(0)}$. Using the Einstein equation $\hat{G}_{M N}=\hat{R}_{M N}-$ $\frac{1}{2} \hat{R} \hat{g}_{M N}=-\Lambda \hat{g}_{M N}+8 \pi G_{5} T_{M N}^{(0)}$, we see for any null vector $\xi^{M}$

$$
\begin{aligned}
T_{M N}^{(0)} \xi^{M} \xi^{N} & =\frac{1}{8 \pi G_{5}} \hat{R}_{M N} \xi^{M} \xi^{N} \\
& =\frac{1}{8 \pi G_{5}}\left\{-\left[A^{\prime \prime}+4\left(A^{\prime}\right)^{2}\right] \xi_{\mu} \xi^{\mu}-4\left[A^{\prime \prime}+\left(A^{\prime}\right)^{2}\right]\left(\xi^{y}\right)^{2}\right\} \\
& =-\frac{3}{8 \pi G_{5}} A^{\prime \prime}\left(\xi^{y}\right)^{2} .
\end{aligned}
$$

On the last line we used $\xi_{M} \xi^{M}=\xi_{\mu} \xi^{\mu}+\left(\xi^{y}\right)^{2}=0$.

Therefore, the WEC for the background matter $T_{M N}^{(0)} \xi^{M} \xi^{N} \geq 0$ for any null vector $\xi^{M}$ becomes equivalent to $A^{\prime \prime} \leq 0$. Subsequently, $A^{\prime}$ must be a non-increasing monotonic function in the coordinate $y$. From the $y y$-component of the Einstein equation, we also find

$$
\Lambda(y)=-6\left(A^{\prime}\right)^{2},
$$

which implies that as one goes to large $y$, the cosmological constant can only become more negative provided that $A^{\prime}$ was a negative value at some point. 


\section{FIGURES}
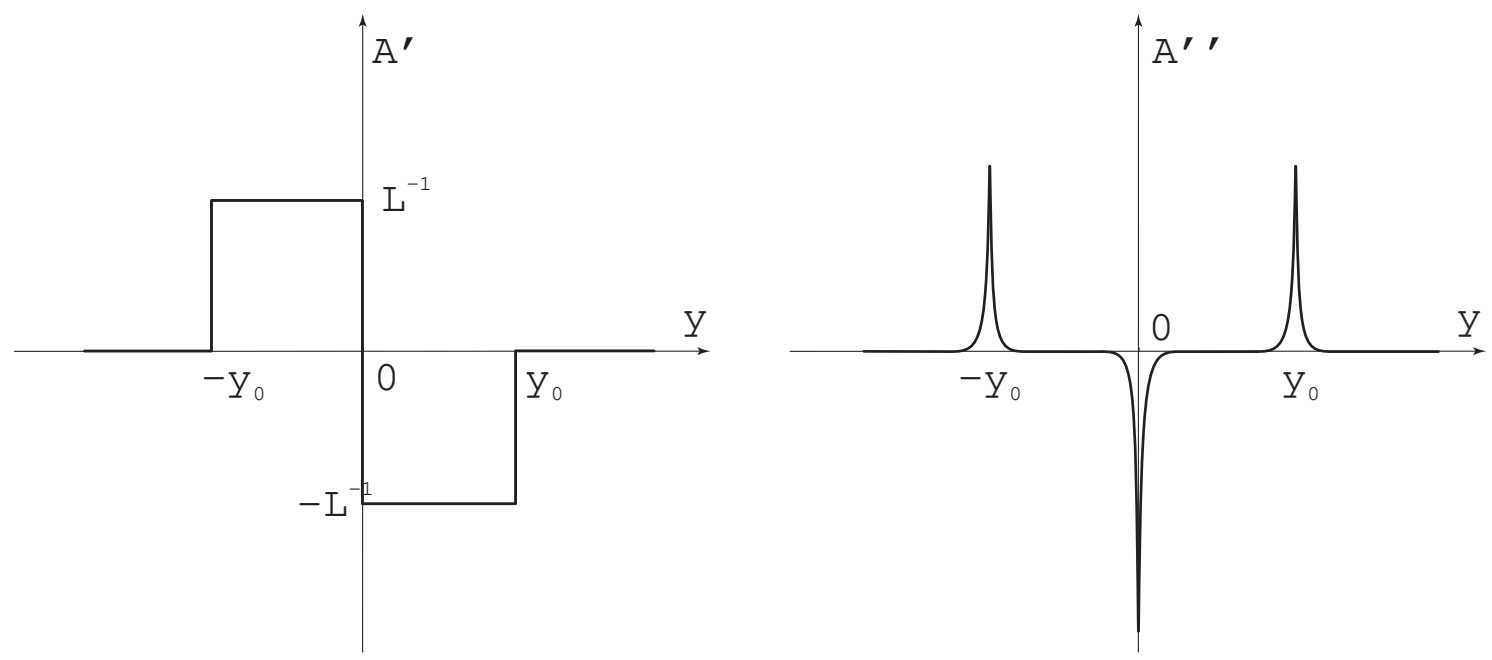

FIG. 1. The behavior of $A^{\prime}$ and $A^{\prime \prime}$

For the perfect $A d S_{5}$ spacetime which corresponds to $\Lambda=$ constant $<0$ and $T_{M N}^{(0)}=0$, $A_{A d S}(y)=-y / L$ where $L$ is the radius of $A d S_{5}$. Thus the perfect anti-de Sitter spacetime leads to $A^{\prime}=-1 / L, A^{\prime \prime}=0$, and so there is no RG flow at all. For the RS spacetime, $A_{R S}(y)=-|y| / L$. Then $A_{R S}^{\prime}(y)=-\theta(y) / L, A_{R S}^{\prime \prime}(y)=-2 \delta(y) / L \leq 0$, and so the RS model satisfies the WEC. For the GRS spacetime, however,

$$
A_{G R S}(y)=\left\{\begin{array}{lll}
-|y| / L & \text { for } & |y| \leq y_{0} \\
-y_{0} / L & \text { for } & |y| \geq y_{0}
\end{array}\right.
$$

Therefore, we have

$$
A_{G R S}^{\prime}(y)=\left\{\begin{array}{ccc}
-\theta(y) / L & \text { for } & |y| \leq y_{0}, \\
0 & \text { for } & |y| \geq y_{0},
\end{array} \quad A_{G R S}^{\prime \prime}(y)=-\frac{2}{L} \delta^{G R S}(y),\right.
$$

where $\delta^{G R S}(y) \equiv \delta(y)-\frac{1}{2} \delta\left(y-y_{0}\right)-\frac{1}{2} \delta\left(y+y_{0}\right)$.

As is shown in Fig. 1, $A_{G R S}^{\prime}(y)$ is not a monotonically decreasing function and thus the condition of $A^{\prime \prime} \leq 0$ does not hold. On the position of the negative branes $\left(y= \pm y_{0}\right)$, one finds $A^{\prime \prime}(y)=\delta\left(y \pm y_{0}\right) / L$. Of course, this indicates the violation of $A^{\prime \prime}(y) \leq 0$. We wish to emphasize that $y= \pm y_{0}$ are just points at which the negative branes are introduced to obtain the Minkowski spacetime for $|y| \geq y_{0}$. In this sense the GRS model can be considered as a regularized version of the RS model. The fact that the GRS model violates the weakest form of a positive energy condition seems to indicate that the GRS background spacetime may be unstable. However, this is not all of the story. Whether or not the GRS background spacetime is really unstable should be checked by the perturbation study, which we shall do below. 


\section{LINEARIZED PERTURBATIONS IN GRS MODEL}

The GRS model with a positive tension domain wall at $z=0$ and two negative tension walls at $z= \pm z_{0}$ [2] perpendicular to the extra fifth direction can be described by the following action:

$$
I=\int d^{4} x \int_{-\infty}^{\infty} d z\left[\frac{1}{16 \pi G_{5}} \sqrt{-\hat{g}}(\hat{R}-2 \Lambda)-\sqrt{-\hat{g}_{B}} \sigma(z)+\mathcal{L}_{M}\right]
$$

Although we used the horospherical coordinates $x^{M}=\left(x^{\mu}, y\right)$ in the previous section, we introduce here the conformally flat coordinates $x^{M}=(x, z)$ for our perturbative analysis. Here $G_{5}$ is the $5 \mathrm{D}$ Newton's constant, $\Lambda$ the bulk cosmological constant of five dimensinal spacetime, $\hat{g}_{B}$ the determinant of the metric describing the brane, and the tension of the branes $\sigma(z)=\sigma \delta^{\mathrm{GRS}}(z)$ with $\sigma=3 / 4 \pi G_{5} L . I_{M}=\int d^{4} x d z \mathcal{L}_{M}$ denotes the matter action, and it contributes only in the linearized level.

If we introduce a conformal factor as follows

$$
d s^{2}=\hat{g}_{M N} d x^{M} d x^{N}=H^{-2} g_{M N} d x^{M} d x^{N},
$$

the field equation becomes [15,11]

$$
\begin{aligned}
& G_{M N}+3 \frac{\nabla_{M} \nabla_{N} H}{H}-3 g_{M N}\left[\frac{\nabla_{P} \nabla^{P} H}{H}-2 \frac{\nabla_{P} H \nabla^{P} H}{H^{2}}\right] \\
& =8 \pi G_{5}\left[-\frac{\Lambda}{8 \pi G_{5} H^{2}} g_{M N}-\frac{\sqrt{-g_{B}}}{\sqrt{-g}} \frac{|H|}{H^{2}} \sigma(z) g_{\mu \nu} \delta_{M}^{\mu} \delta_{N}^{\nu}-\frac{2}{\sqrt{-\hat{g}}} \frac{\delta I_{M}}{\delta \hat{g}^{M N}}\right]
\end{aligned}
$$

with the Einstein tensor $G_{M N}$ constructed from the metric $g_{M N}$. Now it is straightforward to see that, in the absence of matter source except for the domain walls themselves (i.e., $\left.\delta I_{M} / \delta \hat{g}^{M N}=0\right)$, the most general solution having a $4 \mathrm{D}$ Poincaré symmetry is

$$
d s^{2}=H^{-2}(z)\left(\eta_{\mu \nu} d x^{\mu} d x^{\nu}+d z^{2}\right)
$$

where

$$
H(z)=\left\{\begin{array}{ll}
\frac{1}{L}|z|+1, \\
\frac{1}{L}\left|z_{0}\right|+1,
\end{array} \quad \Lambda(z)=\left\{\begin{array}{lll}
-\frac{6}{L^{2}} & \text { for } & |z| \leq z_{0} \\
0 & \text { for } & |z| \geq z_{0}
\end{array}\right.\right.
$$

Let us consider metric fluctuations around this background spacetime as follows 15, 16]:

$$
g_{M N}=\eta_{M N}+h_{M N} .
$$

Defining $\bar{h}_{M N}=h_{M N}-\frac{1}{2} \eta_{M N} h$ where $h=\eta^{M N} h_{M N}$, the linearized perturbation equation of Eq. (9) is

$$
\begin{aligned}
& -\frac{1}{2} \square \bar{h}_{M N}+\partial_{(M} \partial^{P} \bar{h}_{N) P}-\frac{1}{2} \eta_{M N} \partial^{P} \partial^{Q} \bar{h}_{P Q}-\frac{3 \partial^{P} H}{2 H}\left(\partial_{M} h_{N P}+\partial_{N} h_{M P}-\partial_{P} h_{M N}\right) \\
& -3 \eta_{M N}\left[\left(-\frac{\partial^{P} \partial^{Q} H}{H}+2 \frac{\partial^{P} H \partial^{Q} H}{H^{2}}\right) h_{P Q}-\frac{\partial^{Q} H}{H} \partial^{P} \bar{h}_{P Q}\right]-3\left(\frac{\square H}{H}-2 \frac{\partial_{P} H \partial^{P} H}{H^{2}}\right) h_{M N} \\
& +8 \pi G_{5} H^{-2}\left\{\frac{\Lambda(z)}{8 \pi G_{5}} h_{M N}+|H| \sigma(z)\left[\frac{1}{2}\left(\eta^{\alpha \beta} h_{\alpha \beta}-\eta^{P Q} h_{P Q}\right) \eta_{\mu \nu} \delta_{M}^{\mu} \delta_{N}^{\nu}+\delta_{M}^{\mu} \delta_{N}^{\nu} h_{\mu \nu}\right]\right\} \\
= & 8 \pi G_{5} T_{M N},
\end{aligned}
$$


where the linearized matter source $T_{M N}=-\delta\left(2 \delta I_{M} / \sqrt{-\hat{g}} \delta \hat{g}^{M N}\right)$ is included, and $\square=$ $\eta^{M N} \partial_{M} \partial_{N}$. Taking the 5D harmonic gauge condition,

$$
\partial^{M} \bar{h}_{M N}=0 \quad \text { or } \quad \partial^{M} h_{M N}=\frac{1}{2} \partial_{N} h
$$

the linearized equation becomes

$$
\begin{aligned}
& \square h_{M N}+3 \frac{\partial_{5} H}{H}\left(\partial_{M} h_{5 N}+\partial_{N} h_{5 M}-\partial_{5} h_{M N}\right)+\left(-\frac{8\left(\partial_{5} H\right)^{2}}{H^{2}}+\frac{4 \partial_{5}^{2} H}{H}\right) h_{55} \eta_{M N} \\
& -6 \frac{\partial_{5}^{2} H}{H}\left[\frac{1}{2} h_{55} \eta_{\mu \nu} \delta_{M}^{\mu} \delta_{N}^{\nu}-h_{5 \mu}\left(\delta_{M}^{\mu} \delta_{N}^{5}+\delta_{M}^{5} \delta_{N}^{\mu}\right)\right]=-16 \pi G_{5}\left(T_{M N}-\frac{1}{3} \eta_{M N} T_{P}^{P}\right) .
\end{aligned}
$$

Notice that we do not impose the trace free condition $h=h_{M}^{M}=0$. In components, the above equations become

$$
\begin{aligned}
& \left(\square+3 \frac{\partial_{5} H}{H} \partial_{5}\right) h_{55}+\left(-\frac{8\left(\partial_{5} H\right)^{2}}{H^{2}}+\frac{4 \partial_{5}^{2} H}{H}\right) h_{55}=-\frac{32 \pi G_{5}}{3}\left(T_{55}-\frac{1}{2} T_{\rho}^{\rho}\right), \\
& \left(\square+6 \frac{\partial_{5}^{2} H}{H}\right) h_{5 \mu}+3 \frac{\partial_{5} H}{H} \partial_{\mu} h_{55}=-16 \pi G_{5} T_{5 \mu}, \\
& \left(\square-3 \frac{\partial_{5} H}{H} \partial_{5}\right) h_{\mu \nu}+3 \frac{\partial_{5} H}{H}\left(\partial_{\mu} h_{5 \nu}+\partial_{\nu} h_{5 \mu}\right)+\left(-\frac{8\left(\partial_{5} H\right)^{2}}{H^{2}}+\frac{\partial_{5}^{2} H}{H}\right) h_{55} \eta_{\mu \nu} \\
& =-16 \pi G_{5}\left(T_{\mu \nu}-\frac{1}{3} \eta_{\mu \nu} T_{P}^{P}\right) .
\end{aligned}
$$

Note that $\bar{h}=\eta^{M N} \bar{h}_{M N}=-\frac{3}{2} h$.

For simplicity, we consider a case of $h_{5 \mu}=T_{5 \mu}=0$. Then Eq. (17) implies that $h_{55}$ is a function of the $z$-coordinate only. Thus, one can rescale the $z$-coordinate so that $h_{55}=0$. In other words, we take the Gaussian normal gauge (i.e., $h_{5 \mu}=h_{55}=0$ ) without the trace free condition. Subsequently, Eq. (16) shows that it is neccessary for the matter source to satisfy the following relation

$$
T_{55}=\frac{1}{2} T_{\mu}^{\mu}
$$

We see that this is exactly the stabilization condition for the extra dimension implemented in Refs. [17,[18]. Note that $T_{P}^{P}=T_{\mu}^{\mu}+T_{55}=\frac{3}{2} T_{\mu}^{\mu}$. Then Eq. (18) becomes

$$
\left(\square-3 \frac{\partial_{5} H}{H} \partial_{5}\right) h_{\mu \nu}=-16 \pi G_{5}\left(T_{\mu \nu}-\frac{1}{2} \eta_{\mu \nu} T\right),
$$

where $T=T_{\mu}^{\mu}$. Since $h=\eta^{\mu \nu} h_{\mu \nu}+h_{55}=h_{\mu}^{\mu}$, the harmonic gauge condition in Eq. (14) gives

$$
\partial^{\mu}\left(h_{\mu \nu}-\frac{1}{2} \eta_{\mu \nu} h_{\rho}^{\rho}\right)=0, \quad \partial_{5} h_{\rho}^{\rho}=0 .
$$

Since the trace of Eq. (20) is

$$
\left(\square-3 \frac{\partial_{5} H}{H} \partial_{5}\right) h_{\mu}^{\mu}=16 \pi G_{5} T,
$$


Eq. (20) can also be written as

$$
\left(\square-3 \frac{\partial_{5} H}{H} \partial_{5}\right)\left(h_{\mu \nu}-\frac{1}{2} \eta_{\mu \nu} h_{\rho}^{\rho}\right)=-16 \pi G_{5} T_{\mu \nu} .
$$

Thus, the gauge condition in Eq. (21) strictly leads to the source conservation law

$$
\partial^{\mu} T_{\mu \nu}=0
$$

This is a relic of the 4D Poincaré symmetry in the linearized level. Note also that, using the second gauge condition in Eq. (21), we find from Eq. (22)

$$
\square_{4} h_{\mu}^{\mu}=16 \pi G_{5} T_{\mu}^{\mu} \quad \text { with } \quad \square_{4}=\eta^{\mu \nu} \partial_{\mu} \partial_{\nu} .
$$

This means that the trace $h$ can propagate on the brane if one includes the matter source. Note, however this corresponds to the massless scalar propagation. Furthermore, by taking $\partial_{5}$ on Eq. (25) and using $\partial_{5} h_{\mu}^{\mu}=0$, we have additional constraints for the source

$$
\partial_{5} T_{\mu}^{\mu}=\partial_{5} T_{55}=0
$$

Therefore, for the consistency of linearized equations, we find that $T_{\mu}^{\mu}$ and $T_{55}$ of the matter source are to be constant in the extra dimension. Our uniform matter of $T_{\mu}^{\mu}=T_{\mu}^{\mu}(x)$ is lead to keep up the trace $h$ with a physical variable. In the absence of the matter source (more precisely, $T_{\mu}^{\mu}=0$ ), the trace $h$ belongs to a gauge degree of freedom and thus it can be gauged away. In this case, $h$ is not a physical variable. Further our matter source with uniform trace cannot affect the massive KK modes because it is independent of $z$.

Defining $h_{\mu \nu}(x, z)=H^{3 / 2} \psi(z) \hat{h}_{\mu \nu}(x), \psi(z)$ satisfies the following Schrödinger-like equation

$$
\left[-\frac{1}{2} \partial_{5}^{2}+\frac{15\left(\partial_{5} H\right)^{2}}{8 H^{2}}-\frac{3 \partial_{5}^{2} H}{4 H}\right] \psi(z)=\frac{1}{2} m_{h}^{2} \psi(z)
$$

where the seperation constant $m_{h}$ plays as the mass of the $4 \mathrm{D}$ gravitational wave $\hat{h}_{\mu \nu}(x)$. Then Eq. (20) becomes

$$
\left(\square_{4}-m_{h}^{2}\right) h_{\mu \nu}=-16 \pi G_{5}\left(T_{\mu \nu}-\frac{1}{2} \eta_{\mu \nu} T\right),
$$

\section{NO TACHYONIC CONDITION}

Now we are in a position to discuss "no tachyonic condition." Defining $2 \ln H(z)=B(z)$, Eq. (27), which determines the spectrum of KK excitations, can be written as

$$
-\frac{d^{2} \psi(z)}{d z^{2}}+\left[\frac{9}{16} B^{\prime}(z)^{2}-\frac{3}{4} B^{\prime \prime}(z)\right] \psi(z)=m_{h}^{2} \psi(z) .
$$

This can be further taken into the factorization form [3, 19]

$$
\left[-\frac{d}{d z}+\frac{3}{4} B^{\prime}(z)\right]\left[\frac{d}{d z}+\frac{3}{4} B^{\prime}(z)\right] \psi(z)=m_{h}^{2} \psi(z)
$$


which has the form of the supersymmetric quantum mechanics $Q^{\dagger} Q \psi(z)=m_{h}^{2} \psi(z)$, with $Q=\frac{d}{d z}+\frac{3}{4} B^{\prime}(z)$.

The lowest energy state is the zero-energy state which satisfies the supersymmetric condition $Q \hat{\psi}_{0}(z)=0, \hat{\psi}_{0}(z)=e^{-\frac{3}{4} B(z)}=H^{-3 / 2}(z)$. This does not correspond to the normalizable spin-2 propagation. Hence there is no negative energy graviton modes (tachyon modes) in the GRS model. We prove that $m_{h}^{2} \geq 0$.

\section{NO GHOST STATE}

Now we examine the graviton propagator on the positive brane at $z=0$ by considering $h_{\mu \nu}(x, 0) \sim \hat{h}_{\mu \nu}(x)$ only. It requires the bilinear forms of the source with the inverse propagator to isolate the physical modes. As the present analysis is on the classical level, we express $\hat{h}_{\mu \nu}$ in terms of source. Taking Fourier transformation for Eq. (28) to momentum space results in

$$
\hat{h}_{\mu \nu}(p)=\frac{16 \pi G_{5}}{p^{2}+m_{h}^{2}}\left[T_{\mu \nu}(p)-\frac{1}{2} \eta_{\mu \nu} T(p)\right] .
$$

Then the one graviton exchange amplitude for the source $T_{\mu \nu}$ is given by [16, 18]

$$
A^{\text {class }}=\frac{1}{4} \hat{h}_{\mu \nu}(p) T^{\mu \nu}(p)=\frac{4 \pi G_{5}}{p^{2}+m_{h}^{2}}\left(T^{\mu \nu} T_{\mu \nu}-\frac{1}{2} T^{2}\right) .
$$

In order to study the massive states, it is best to use the rest frame [20] in which

$$
p_{1} \neq 0, \quad p_{2}=p_{3}=p_{4}=0 .
$$

Considering Eqs. (24) and (33) leads to the following source relations

$$
T_{11}=T_{12}=T_{13}=T_{14}=0 .
$$

Thus, one obtains

$$
T^{\mu \nu} T_{\mu \nu}-\frac{1}{2} T^{2}=\left|T_{+2}\right|^{2}+\left|T_{-2}\right|^{2}+\left|T_{+1}\right|^{2}+\left|T_{-1}\right|^{2}+T_{44}\left[\frac{1}{2} T_{44}-\left(T_{22}+T_{33}\right)\right],
$$

where the first two terms correspond to the exchange of graviton with helicity- $2 T_{ \pm 2}=$ $\frac{1}{2}\left(T_{22}-T_{33}\right) \pm i T_{23}$, and the third and fourth terms are the exchange of the graviphoton with helicity-1 $T_{ \pm 1}=T_{24} \pm i T_{34}$. We note here that the last term in the above equation is not positive definite. This means that there exist ghost states (negative norm states) in general. However, if one requires

$$
T_{44}=2\left(T_{22}+T_{33}\right)
$$

one immediately finds that

$$
T^{\mu \nu} T_{\mu \nu}-\frac{1}{2} T^{2}=\left|T_{+2}\right|^{2}+\left|T_{-2}\right|^{2}+\left|T_{+1}\right|^{2}+\left|T_{-1}\right|^{2}
$$


with all positive norm states and without helicity-0 states (graviscalars). In the case of $2\left(T_{22}+T_{33}\right)=a T_{44}$ with $a<1$, we find no ghost states, but there exist the graviscalars which arise from the diagonal elements of $T_{\mu \nu}$.

In the limit of $m_{h}^{2} \rightarrow 0$, the graviphoton propagation can be decoupled from the brane [21]. Hence we can neglect $\left|T_{ \pm 1}\right|^{2}$-terms. Finally the amplitude takes the form

$$
A_{m_{h}^{2} \rightarrow 0}^{\text {class }}=\lim _{m_{h}^{2} \rightarrow 0} \frac{4 \pi G_{5}}{p_{1}^{2}+m_{h}^{2}}\left[\left|T_{+2}\right|^{2}+\left|T_{-2}\right|^{2}\right],
$$

which corresponds to the massless spin-2 amplitude.

\section{DISCUSSION}

Naively, it is conjectured that, if a certain $A d S_{5}$ spacetime does not satisfy the WEC, this may belong to an unstable manifold. However, this is not all of the story. We believe that the stability analysis of the given spacetime in curved space is based on the perturbation study around the background spacetime. This corresponds to testing the reliability of its linearized approximation. Hence we investigate the stability of the GRS spacetime along this line. Here, as in usual Minkowski background, we require two conditions for the stable background: no tachyon and no ghost states. Especially, we guarantee that the GRS background is stable against the small perturbation because it has no tachyon and no ghost states. No tachyon condition is easily checked by observing the Shrödinger equation for the massive KK modes. However, showing that there is no ghost states in the GRS model is a non-trivial task. This is because, in this model, the massless spin-2 propagation (graviton) can be only described by the massive KK modes with resonance.

The main problem is to cancel the unwanted extra polarization in the quasi-localization of $4 \mathrm{D}$ gravity. This is done by introducing both the trace $(h)$ and the matter source with uniform trace $\left(T_{\mu}^{\mu}\right)$ at the linearized level. In the conventional RS approach, the trace $(h)$ is just a gauge-dependent scalar and hence it can be gauged away. However, including the matter with uniform trace, this plays a role of $\xi^{5}$ in the brane-bending model with the localized source [12]. This is because $h\left(\xi^{5}\right)$ satisfy the nearly same massless equations of $\square_{4} h=16 \pi G_{5} T_{\mu}^{\mu}\left(\square_{4} \xi^{5}=\frac{8 \pi G_{5}}{6} S_{\mu}^{\mu}\right.$ in Ref. [12]). And the comparison of $\bar{h}_{\mu \nu}=h_{\mu \nu}-\frac{1}{2} \eta_{\mu \nu} h$

with $\bar{h}_{\mu \nu}=h_{\mu \nu}^{(m)}+2 L^{-1} \eta_{\mu \nu} \xi^{5}$ in Ref. [12 confirms the close relationship between $h$ and $\xi^{5}$. If $T_{\mu}^{\mu}=0$, one finds from Eq. (32) that the massive spin-2 states have 5 polarizations with all positive norm states [16]. Here, in the case of $h \neq 0, T_{\mu}^{\mu} \neq 0$, requiring the additional condition $T_{44}=2\left(T_{22}+T_{33}\right)$ in Eq. (36), we find the massless spin-2 state with 2 polarizations in the limit of $m_{h}^{2} \rightarrow 0$. In this case the ghost states disappear.

In conclusion, it turns out that the GRS spacetime is stable against the small perturbation if $T_{\mu}^{\mu} \neq 0, h \neq 0$ with $T_{\mu}^{\mu}=2 T_{55}=3\left(T_{22}+T_{33}\right)$. On the other hand, the RS spacetime is stable under the RS gauge $\left(h=h_{5 \mu}=h_{55}=0, \partial_{\mu} h^{\mu \nu}=0\right)$ and $T_{\mu}^{\mu}=0$ [16].

\section{ACKNOWLEDGMENTS}

The authors thank Hyungwon Lee for helpful discussions. This work was supported by the Brain Korea 21 Programme, Ministry of Education, Project No. D-0025. 


\section{REFERENCES}

[1] L. Randall and R. Sundrum, Phys.Rev. Lett. 83, 4690 (1999), hep-th/9906064, Phys.Rev. Lett. 83, 3370 (1999), hep-ph/9905221; N. Arkani-Hamed, S. Dimopoulos, G. Dvali and N. Kaloper, Phys. Rev. Lett. 84, 586 (2000), hep-th/9907209; K. Akama, hep-th/0001113.

[2] R. Gregory, V.A. Rubakov and S.M. Sibiryakov; "Opening up Extra Dimensions at Ultralarge Scales," hep-th/0002072.

[3] C. Csáki, J. Erlich and T.J. Hollowood; "Quasi-Localization of Gravity by Resonant Modes," hep-th/0002161.

[4] G. Dvali, G. Gabadadze and M. Porrati; "Metastable Gravitons and Infinite Volume Extra Dimensions," hep-th/0002190.

[5] E. Witten;"The Cosmological Constant from the Viewpoint of String Theory," hepph/0002297.

[6] C. Csáki, J. Erlich and T.J. Hollowood; "Graviton Propagators, Brane Bending and Bending of Light in Theories with Qusi-Localized Gravity," hep-th/0003020.

[7] R. Gregory, V.A. Rubakov and S.M. Sibiryakovi, hep-th/0003045.

[8] C. Csáki, J. Erlich, T.J. Hollowood and J. Terning; "Holographic RG and Cosmology in Theories with Quasi-Localized Gravity," hep-th/0003076.

[9] G. Dvali, G. Gabadadze and M. Porrati;"A Comment on Brane Bending and Ghosts in Theories with Infinite Extra Dimensions," hep-th/0003054; "4D Gravity on a Brane in 5D Minkowski Space," hep-th/0005016.

[10] L. Pilo, R. Rattazzi and A. Zaffaroni, hep-th/0004028.

[11] G. Kang and Y.S. Myung;"No Ghost State in the Brane World," hep-th/0003162.

[12] J. Garriga and T. Tanaka, Phys. Rev. Lett. 84, 2778 (2000), hep-th/9911055; S. Giddings, E. Katz and L. Randall, JHEP 0003, 023 (2000), hep-th/0002091.

[13] D. Bill, in Spacetime and Geometry, edited by R.A. Matzner and L.C. Sheply (Texas Univ. Press, Austin, 1982).

[14] D.Z. Freedmann, S.S. Gubser, K. Rilch, and N.P. Warner, hep-th/9904017.

[15] M.G. Ivanov and I.V. Volovich;"Metric Fluctuations in Brane Worlds," hep-th/9912242; Y.S. Myung and G. Kang; "Comment on "Metric Fluctuations in Brane Worlds", hepth/0001003.

[16] Y.S. Myung, G. Kang and H.W. Lee, Phys. Lett. B478, 294 (2000), hep-th/0001107.

[17] P. Kanti, I. Kogan, K. Olive and M. Pospelov, Phys. Rev. D61, 106004 (2000), hepph/9912266.

[18] I. Kogan and G. Ross; "Brane Universe and Multigravity: Modification of Gravity at Large and Small Distances," hep-th/0003074.

[19] O. DeWolfe, D.Z. Freedman, S.S. Gubser, and A. Karch; "Modeling the Fifth Dimension with Scalar and Gravity," hep-th/9909134.

[20] A. Salam and J. Strathdee, "On Kaluza-Klein Theory," ICTP preprint, IC/81-211; S. Randjbar-Daemi, A. Salam, and J. Strathdee, Nucl. Phys. B214, 491 (1983); Y.S. Myung and B.H. Cho, Phys. Lett. A111, 412 (1985); Phys. Lett. B166, 75 (1986).

[21] H. van Dam and M. Veltman, Nucl. Phys. B22, 397 (1970); V.I. Zakharov, JETP Lett. 12, $3112(1970)$. 